\title{
A salient distractor does not disrupt conjunction search
}

\author{
DOMINIQUE LAMY and YEHOSHUA TSAL \\ Tel Aviv University, Tel Aviv, Israel
}

\begin{abstract}
Leading theories of attention posit that bottom-up and top-down factors simultaneously affect attentional priority in visual search. Recent evidence, however, suggests that subjects may rely exclusively on top-down guidance when searching for a target defined by a specific known feature (Bacon \& Egeth, 1994). In the present experiment, we addressed this issue in a conjunction search task. We investigated how searching for a green $O$ among green $T s$ and red $O$ is affected by the presence of a distractor with a unique shape (green $\mathrm{X}$ ), color (blue $\mathrm{O}$ ), or both (blue X). We showed that the salient distractor does not disrupt performance on target-present trials, but produces a large interference on target-absent trials. We conclude that salience-based and top-down processes are alternative modes of guidance, rather than joint contributors in the allocation of attentional priority.
\end{abstract}

It is widely agreed that perceptual analysis of the visual world takes place in two successive stages: a stage of preliminary analysis (preattentive stage) that is parallel and operates without capacity limitations, and a stage of more detailed analysis (focal attention) that is serial and operates only on selected parts of the visual field. Two sources of control guide attention at the preattentive stage. Goal-directed or top-down control of attention refers to the ability of the observer's goals or intentions to determine which areas, attributes, or objects will be selected for further visual processing. Stimulus-driven or bottom-up control refers to the capacity of certain stimulus properties to attract attention.

Leading models of visual search assume that top-down and bottom-up factors interact to determine attentional priority (see Lamy \& Tsal, 1998, for a broader discussion). The Guided Search model (Cave \& Wolfe, 1990; Wolfe, 1994) and Treisman and Sato's (1990) revised feature integration theory posit that an item's overall level of attentional priority is the sum of its bottom-up activation level and its top-down activation level. Bottom-up activation is a measure of how different an item is from its neighbors. ${ }^{1}$ Top-down activation (Cave \& Wolfe, 1990), or inhibition (Treisman \& Sato, 1990), depends on the degree of match between an item and the set of target properties specified by task demands.

According to Duncan and Humphreys's $(1989,1992)$ attentional engagement theory, two factors combine to determine selection weights. The first is the extent to which an item's properties match target properties as specified by the task. This is equivalent to the top-down activation described above. The second is weight linkage, which allows the observer to select or reject (spreading suppres-

Correspondence concerning this article should be addressed to D. Lamy, Department of Psychology, Tel Aviv University, Ramat Aviv, Tel Aviv, 69978, Israel (e-mail: domi@freud.tau.ac.il). sion) similar elements together. Weight linkage should be distinguished from the bottom-up component of other models because its contribution is dependent on topdown factors. Indeed, weight linkage does not imply a bias against selecting an item because it is similar to many other items in the display; rather, it only amplifies the effects of top-down factors. This difference is illustrated by the fact that when target-distractor similarity is low (low top-down activation of distractors), distractor heterogeneity does not affect search performance (no effect of weight linkage).

These theories differ in the extent to which they consider effects of salience to depend on task demands. However, they all predict that a salient distractor will be disruptive to search performance, either because it has a high level of bottom-up activation (e.g., Cave \& Wolfe, 1990; Treisman \& Sato, 1990) or because it suffers less from spreading suppression than a distractor that is not salient (e.g., Duncan \& Humphreys, 1989, p. 456).

In the last few years, a considerable amount of research has been devoted to the investigation of such salience effects. In these studies, salient distractors were always singletons on various dimensions (such as color, e.g., Bacon \& Egeth, 1994; luminance, e.g., Theeuwes, 1992; or shape, e.g., Pashler, 1988). These studies have led to two major conclusions. First, when attention is directed by advance knowledge of target location, no salient distractor of any kind will capture attention (Theeuwes, 1991; Yantis \& Jonides, 1990). Second, when the target is defined by a known nonspatial attribute, such as its color (feature-search tasks), salient items on an irrelevant dimension will capture attention only if subjects adopt the strategy of searching for an odd item (e.g., Bacon \& Egeth, Experiment 1; Pashler, 1988; Theeuwes, 1992; see Folk, Remington, \& Johnston, 1992, and Yantis \& Egeth, in press, for further elaboration on this idea) instead of searching for the target feature (singleton-detection mode vs. feature-search mode; Pashler, 1988). For instance, Bacon and Egeth (1994, Experiments 2 and 3 ) showed that when the singleton- 
detection mode of search is discouraged, a singleton on an irrelevant dimension does not interfere with feature search. They also proposed that when both strategies can be efficiently used to perform the task, the singletondetection mode is preferred (Experiment 1), possibly because it is less cognitively demanding than the featuresearch mode.

In summary, the results of these studies cannot be accommodated within the framework of visual search theories that assume the joint contribution of top-down and bottom-up factors to attentional priority. Instead, they suggest that in feature search tasks, subjects may use the feature-search mode, thus relying exclusively on topdown guidance.

Numerous studies have investigated whether or not salience affects performance on spatial cuing and feature search tasks, but no study to date has addressed this question with conjunction search tasks. This issue is especially important because the theories that assume that both top-down factors and salience contribute to attentional priority focus their explanatory efforts on conjunction search tasks (e.g., Cave \& Wolfe, 1990; Duncan \& Humphreys, 1989; Treisman \& Sato, 1990); moreover, they often posit that feature search and conjunction search are subserved by qualitatively different processes (e.g., Treisman, 1991), so that conclusions drawn from feature search task studies may not extend to conjunction search tasks. However, no experiment has actually tested the hypothesis that salience necessarily affects conjunction search, because the manipulations used so far have not isolated salience effects from the effects of top-down factors. Indeed, studies that have manipulated targetdistractor similarity (e.g., Cave \& Wolfe, 1990; Duncan \& Humphreys, 1989; Treisman \& Gormican, 1988) have revealed only the effects of top-down factors. They have typically shown that it is easier to look for a red $\mathrm{O}$ among green $O$ s and red $\mathrm{Ts}$, for instance, than for a red $\mathrm{O}$ among orange Os and red Ts. This manipulation reduces target salience by the same amount as each distractor's salience. Therefore, the relative salience of each item in the display remains unchanged. Similarly, studies that have manipulated distractor-distractor similarity (e.g., Duncan \& Humphreys, 1989) have typically shown that it is easier to look for a target among homogeneous than among heterogeneous distractors. However, this result may not necessarily be due to an increase in distractors' salience relative to that of the target. Increased distractor heterogeneity usually heightens the probability that some distractors will be similar to the target (Tsal, Meiran, \& Lamy, 1995). This idea is consistent with the finding that distractor-distractor similarity has no effect on search efficiency when all distractors are dissimilar to the target (Duncan \& Humphreys, 1989). Distractor heterogeneity effects may therefore result only from top-down factors.

The objective of the present experiment was to investigate the effects of salience on conjunction search performance by using a manipulation that affects the rela- tive salience of the target while leaving top-down activation level unchanged.

We used a typical conjunction search task in which subjects had to look for a green $O$ (the target) among green Ts and red Os (conjunction distractors) in displays of variable sizes. The critical manipulation was to replace one of the conjunction distractors by a salient distractor on part of the trials. The salient distractor could be unique on the shape dimension (green X) or on the color dimension (blue $\mathrm{O}$ ). It was highly salient because it was the only element with a unique feature in the display, and its presence thus notably modified the distribution of bottom-up activations in the display. However, its level of top-down activation was the same as that of the conjunction distractor it replaced, because it shared only one feature with the target (green or O, respectively) and was equally dissimilar from it on the other dimension ( $X$ and $\mathrm{T}$ vs. $\mathrm{O}$, and blue and red vs. green, respectively). The salient distractor could also be unique on both dimensions (blue $\mathrm{X}$ ), in which case it received maximum bottom-up activation, but no top-down activation.

In the present task, as in Bacon and Egeth's task (1994, Experiments 2 and 3), relying on a salience-based strategy was clearly detrimental, because the target was never the most salient item. If bottom-up activation affects conjunction search regardless of task requirements, performance should be disrupted by the presence of the salient distractor. If, in contrast, subjects are able to rely exclusively on a top-down strategy, performance should be comparable whether or not a salient distractor is present.

\section{METHOD}

\section{Subjects}

Subjects were 12 Tel Aviv University undergraduates, who participated in the experiment for a course credit. All reported having normal or corrected-to-normal visual acuity and normal color vision.

\section{Apparatus}

Displays were generated by an IBM-PC-compatible computer attached to a VGA color monitor, using $640 \times 480$ graphics mode. Responses were collected via the computer keyboard.

\section{Stimuli}

The fixation display was a white $0.1^{\circ} \times 0.1^{\circ}$ plus sign $(+)$ in the center of a black background. Stimulus displays consisted of 6,8 , or 10 colored letters equally spaced along the circumference of an imaginary circle centered at fixation. Display density thus increased with number of letters. At a viewing distance of $50 \mathrm{~cm}$, the centers of the letters were $3.91^{\circ}$ from fixation and each letter was $0.69^{\circ}$ wide $\times 1.04^{\circ}$ high. The target was always a green O. Conjunction distractors were red $O s$ and green Ts. The salient distractor was a green $X$ (salient shape), a blue $\mathrm{O}$ (salient color), or a bluc $X$ (salient shape and color). There were four possible conditions: (1) the target-present and salient-distractor-present condition, (2) the target-present and salient-distractor-absent condition, (3) the target-absent and salient-distractor-present condition, and (4) the target-absent and salient-distractor-absent condition. On trials in which both the target and the salient distractor were absent, the display consisted of an equal number of each type of conjunction distractor. When present, the target and salient-distractor each replaced 
one, randomly selected conjunction distractor. Letter positions were assigned randomly, with the constraint that three letters with the same color or shape were never adjacent. CIE coordinates and luminance values for stimuli were as follows: green, $x=.284, y=$ .580, lum $=19.9 \mathrm{~cd} / \mathrm{m}^{2} ;$ red, $x=.582, y=.336$, lum $=12.3 \mathrm{~cd} / \mathrm{m}^{2}$ and biue, $x=.192, y=.223$, lum $=9.8 \mathrm{~cd} / \mathrm{m}^{2}$. Figure 1 shows $\mathrm{ex}$ amples of the stimulus display for each condition of target presence $\times$ salient-distractor presence.

\section{Procedure}

Subjects responded with a keypress. They indicated target presence with their dominant hand ("l" on the numerical keypad for right-handed subjects and on the number strip for left-handed subjects) and target absence with their other hand (" 3 " on the number strip for right-handed subjects and on the numerical keypad for lefthanded subjects). They were instructed to respond as fast as possible while maintaining very high accuracy. The subjects were verbally informed of the identity and color of the target letter. They were told that nontargets were letters of different shapes and colors in variable number. Eye movements were not monitored, but subjects were explicitly requested to maintain fixation throughout each trial. Trial presentation was forced paced. The fixation display was pre- sented for $350 \mathrm{msec}$ and was followed by the target display, which remained visible for $2,500 \mathrm{msec}$ or until response. The screen went blank for $1,500 \mathrm{msec}$ before the next trial began. A $500-\mathrm{msec}$ feedback beep was sounded whenever a response error was recorded.

After subjects heard the instructions, they were given 36 practice trials randomly selected from the experimental trials. There were two blocks of 144 experimental trials. Subjects were allowed a rest period between them. The relevant factors (target presence, salientdistractor presence, set size) resulted in 12 equally probable conditions $(2 \times 2 \times 3)$. Within the salient-distractor-present condition, each of the three possible salient distractor types was equally probable. The order of the trials was randomized by computer for each subject.

\section{RESULTS}

Reaction times (RTs) greater than $1,500 \mathrm{msec}$ were removed from the analysis $(0.8 \%$ of trials). The mean hit rate on target-present trials was $97.5 \%$ and the mean false alarm rate on target-absent trials was $0.8 \%$. An analysis of variance (ANOVA) was performed on correct RTs with

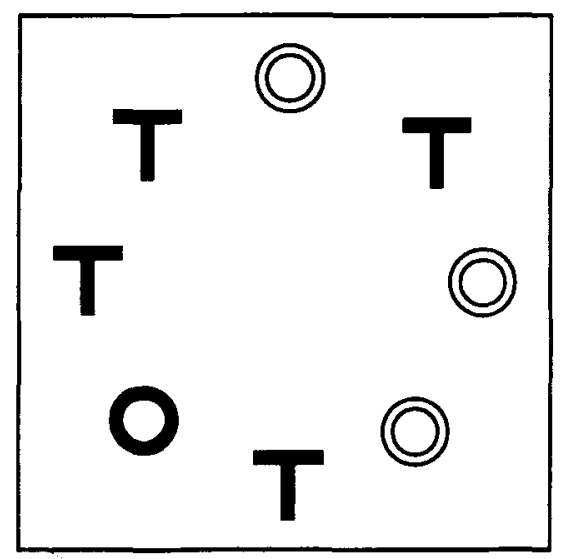

Target-present / Distractor Absent

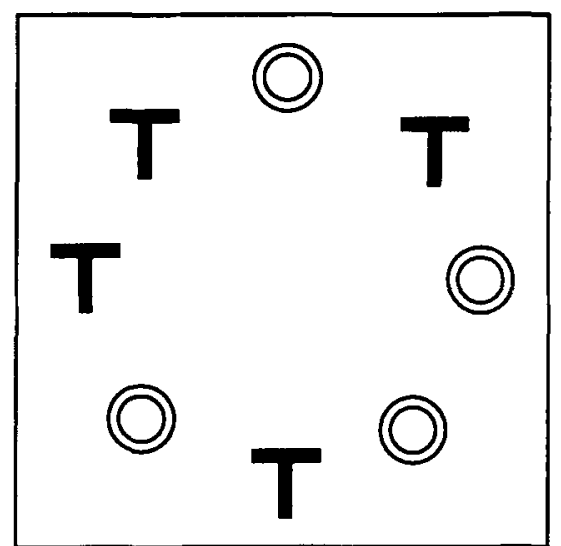

Target-Absent / Distractor Absent

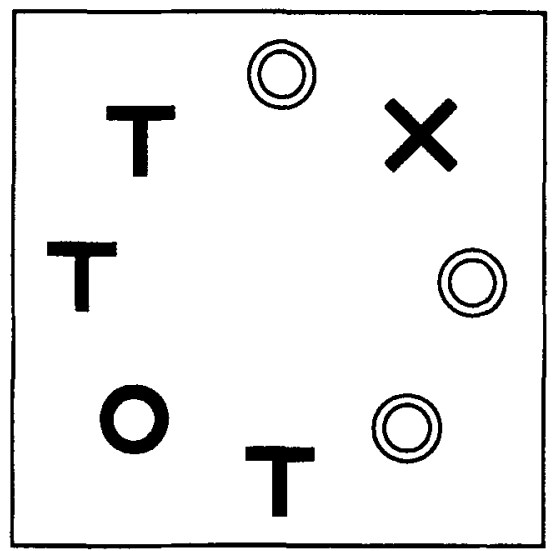

Target-present / Distractor Present

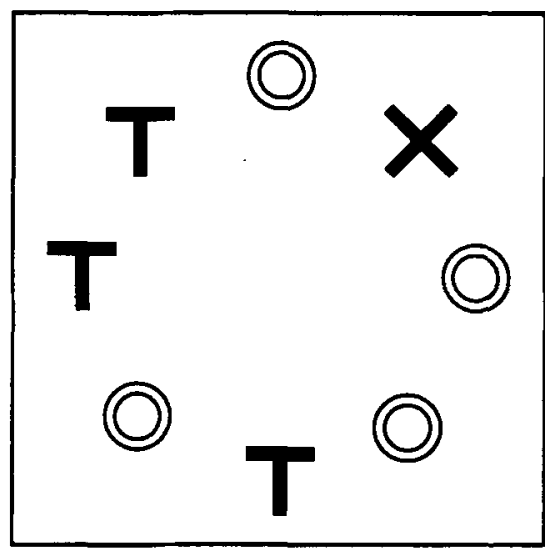

Target-Absent / Distractor Present

Figure 1. Sample stimulus displays (with display size 8). Upper panels show the target-present conditions, in which a green $O$ target appears among green Ts and red $O s$, and lower panels show the target-absent conditions. Right panels show the salient-distractor-present conditions in which the salient distractor is a green $X$, and left panels show the salient-distractor-absent condition. (White letters were red; black letters were green). 
target presence (target-present vs. target-absent), salientdistractor presence (salient-distractor-present vs. salientdistractor-absent), and display size $(6,8,10)$ as withinsubjects factors. Table 1 presents the subjects' average RTs in the 12 different conditions. Responses were faster on target-present than on target-absent trials [619 vs. $774 \mathrm{msec} ; F(2,10)=46.6, p<.0001]$ and on salientdistractor-absent than on salient-distractor-present trials [685 vs. $708 \mathrm{msec} ; F(1,11)=12.1, p<.006]$. The main effect of display size was also highly significant $[F(2,10)$ $=22.9, p<.0002]$, with an average slope of $18.5 \mathrm{msec}$ per item. Most importantly, the interaction of target presence and salient-distractor presence was highly significant $[F(1,11)=10.6, p<.008]$. The analysis of simple effects clarified this interaction. The presence of a salient distractor considerably slowed response on target-absent trials [ $796 \mathrm{msec}$ on salient-distractor-present trials vs. $752 \mathrm{msec}$ on salient-distractor-absent trials; $F(1,11)=$ $17.1, p<.002]$, but this effect disappeared on targetpresent trials $[621 \mathrm{msec}$ vs. $617 \mathrm{msec} ; F(1,11)=0.31$, $p>.5]$. No other interaction reached significance.

Because mean RTs were significantly faster in the target-present than in the target-absent condition, one might argue that the effect of salient-distractor presence was not revealed in the fast target-present responses, but became large enough in the slower target-absent responses. In order to rule out this possibility, we equated the average RTs in target-present and target-absent conditions by conducting a separate analysis on the $50 \%$ fastest trials in the target-absent condition, separately for each condition of salient-distractor presence, for each subject. With this procedure, the average RT in the target-absent condition became $648 \mathrm{msec},{ }^{2}$ which was comparable to the 620-msec average RT in the target-present condition. In the target-absent condition, the effect of salient-distractor presence remained highly significant $[t(11)=4.14, p<$ $.005]$, with this difference being in the right direction for 11 of the 12 subjects. The interaction of target presence and salient-distractor presence can therefore not be attributed to overall differences in mean RTs between target-present and target-absent conditions.

In order to measure the effects of salient distractor type, an ANOVA was performed on correct target-absent trials in which a salient distractor was present, with distractor type (salient shape, salient color, salient shape and color) and display size as factors. The main effect of salient distractor type did not reach significance $[F(2,10)=$
$1.1, p>.3]$. The interaction between the two factors was not significant.

\section{DISCUSSION}

The main finding of the present experiment was that the presence of a salient distractor did not disrupt performance on target-present trials in a conjunction search task. This result is in line with previous findings using feature search tasks (Bacon \& Egeth, 1994). However, it stands in contrast with a basic tenet of several leading theories of attention (e.g., Cave \& Wolfe, 1990; Treisman \& Sato, 1990; Wolfe, 1994), namely that top-down and bottom-up factors combine to guide selection. Although the newer version of the Guided Search model (Wolfe, 1994) does assume that the relative weight of bottom-up factors is modulated by task demands, it still contends that it is never reduced to zero (p. 209). In contrast with such claims, the present results suggest that subjects may rely exclusively on a top-down strategy in order to perform conjunction as well as feature search tasks, with salience playing no role whatsoever.

There are a number of potential criticisms concerning this finding because it is based on a null effect. First, one might argue that because search was serial (with a slope of $17.3 \mathrm{msec} /$ item on target-present trials), letters may have been selected randomly (i.e., without any guidance), thus explaining the absence of salience effects (Bacon \& Egeth, 1994; Theeuwes, 1991). However, although the average slope found here was significantly greater than zero, the slopes of strictly serial searches are typically much steeper (e.g., Wolfe, Cave, \& Franzel, 1989). In addition, a comparable search slope was found on target-absent trials (19.7 msec/item), in which large distraction effects were observed.

Second, because of the particular feature values that were chosen for the salient distractor, on the one hand, and for the conjunction distractors, on the other hand, the salient distractor may have had a lower level of topdown activation than did conjunction distractors. This may have happened if the features of the conjunction distractor that were not shared by the target (red and $\mathrm{T}$ ) were always more similar to the target features (green and $\mathrm{O}$ ) than were the features of the salient distractor (blue and T). As a result, some bottom-up activation may have accrued to the salient distractor, but it may have been too small to offset the difference in top-down activation be-

Table 1

Mean Correct RTs (in Milliseconds) by Display Size for

Target-Present Versus Target-Absent Conditions and Salient-Distractor-Present Versus Salient-Distractor-Absent Conditions

\begin{tabular}{|c|c|c|c|c|c|c|}
\hline \multirow[b]{2}{*}{ Condition } & \multicolumn{2}{|c|}{6 Letters } & \multicolumn{2}{|c|}{8 Letters } & \multicolumn{2}{|c|}{10 Letters } \\
\hline & Target & No target & Target & No target & Target & No target \\
\hline Salient Distractor & 588 & 747 & 620 & 807 & 656 & 838 \\
\hline No Salient Distractor & 578 & 715 & 629 & 756 & 644 & 787 \\
\hline
\end{tabular}


tween the salient distractor and the other distractors. Again, this is unlikely because the same conditions prevailed in the target-absent condition, in which distraction effects were observed.

Third, top-down activation is likely to have been high in the present task because target and distractor features were highly discriminable. As a consequence, one might argue that the present finding only indicates that topdown activation may override bottom-up activation, but does not support the claim that there was no bottom-up component altogether in the calculation of attentional priority levels. The argument goes as follows. If the topdown activation contributed by each target feature was much higher than the bottom-up activation generated by the salient distractor's unique feature, then the target may have always been selected before the salient distractor, leaving no opportunity for the latter to interfere with performance. However, the salient distractor had the same top-down activation level as any conjunction distractor because it also shared a feature with the target, but a higher bottom-up activation level because it had a unique feature. In order to support the idea that salience did affect attentional priority but that a ceiling effect masked salience effects, one must assume that no distractor at all was ever selected before the target-that is, that search was strictly parallel. The significant display size effect argues against this possibility. Thus, we can conclude that salience did not contribute to guide selection.

The second relevant finding of the present experiment concerns target-absent trials. On such trials, a large effect of salient-distractor presence was observed. It is difficult to relate this finding to the existing literature because in studies that used the present rationale with feature search tasks, subjects had to either identify (e.g., Bacon \& Egeth, 1994; Theeuwes, 1992) or localize (Pashler, 1988) the target, rather than simply detect it. Consequently, there were no target-absent trials.

We argue that the disruption caused by the salient distractor on target-absent trials must have resulted from postselective processes. First, any factor impeding selection in the present task should have affected performance on target-present trials and on target-absent trials to a comparable extent. The overall activation level of each type of distractor was different in the two types of trials because in target-absent trials the target did not participate in the competition, whereas in target-present trials, it attracted a substantial amount of attentional resources. However, the ranking of the salient distractor relative to conjunction distractors in the activation ladder (which is the result of preattentive guidance by top-down factors, bottom-up factors, or both) remained the same on targetpresent trials as on target-absent trials. Because we found that the salient distractor was equally likely as any conjunction distractor to be selected on target-present trials, it follows that it was so also on target-absent trials. Thus, we can conclude that salience affected preattentive guidance on neither type of trial. Second, the greater variability generally observed on target-absent trials relative to target-present trials (e.g., Cave \& Wolfe, 1990, p. 264) was also found in the present study. Target-present slopes ranged from 9 to $34.5 \mathrm{msec} /$ item, whereas target-absent slopes ranged from 1.5 to $42.7 \mathrm{msec}$. This greater variability on target-absent trials suggests that subjects use different strategies to decide when to terminate their search on target-absent trials, which is consistent with the idea that a factor that affects only target-absent trials results from processes other than selection.

At this point, one can only speculate as to what postselective factor might explain why the salient distractor slowed responses. One possibility is that maintaining a state of directed attention (i.e., channeling attention toward a certain location or feature) may be effortful. Thus, when subjects terminate the search after a certain floor level is reached down the ladder of activation in the search for the target (Wolfe, 1994), they may regress to a less demanding, distributed mode of attention as a last checking procedure. Because salient distractors are able to capture attention when subjects are in a distributed or unfocused attentional state (see, e.g., Theeuwes, 1991), this may account for the delay of "no" responses observed on salient-distractor-present trials. A second possibility is that subjects may have been more reluctant to emit a negative response after analyzing the salient distractor because their experience of the task had taught them to expect $\mathrm{T}$ and "red" as nontarget features, rather than $\mathrm{X}$ or "blue."

To conclude, the present finding strongly suggests that subjects may rely exclusively on a top-down strategy in order to perform conjunction search tasks, with bottomup activation exerting no effect. We found that the presence of a salient distractor did not affect search on targetpresent trials and did so only on target-absent trials. We explained why the latter effect is most likely to have resulted from some postselective process rather than having taken place during selection. Because we proposed only post hoc suggestions of what postselective factor may have been at play, there remains a small possibility that, by the same token, some weak bottom-up activation was present in the search but, for reasons yet to be elucidated, it was revealed only on target-absent trials. The important point, however, is that a central hypothesis of leading theories of attention (see, e.g., Cave \& Wolfe, 1990; Treisman \& Sato, 1990) posits that a bottom-up mechanism always activates items with unique features regardless of the task, but (1) it is only an assumption, since it has not been tested so far, and (2) our finding considerably weakens it. Thus, the present study may at least point to the need for more empirical efforts to be devoted to this issue and for existing theories of attention to be reevaluated accordingly.

\section{REFERENCES}

BaCON, W. F., \& EgETH, H. E. (1994). Overriding stimulus-driven attentional capture. Perception \& Psychophysics, 55, 485-496.

Cave, K. R., \& WolfE, J. M. (1990). Modelling the role of parallel processing in visual search. Cognitive Psychology, 22, 225-271. 
DunCan, J., \& Humphreys, G. W. (1989). Visual search and stimulus similarity. Psychological Review, 96, 433-458.

Duncan, J., \& Humphreys, G. W. (1992). Beyond the search surface: Visual search and attentional engagement. Journal of Experimental Psychology: Human Perception \& Performance, 18, 578-588.

Folk, C. L., REMington, R. W., \& Johnston, J. C. (1992), Involuntary covert orienting is contingent on attentional control settings. Journal of Experimental Psychology: Human Perception \& Performance, 18, 1030-1044.

LAMY, D., \& TSAL, Y. (1998). On the status of location in visual attention. Manuscript submitted for publication.

PASHLER, H. (1988). Cross-dimensional interaction and texture segregation. Perception \& Psychophysics, 43, 307-318.

Theeuwes, J. (1991). Cross-dimensional perceptual selectivity. Perception \& Psychophysics, 50, 184-193.

Theeuwes, I. (1992). Perceptual selectivity for color and form. Perception \& Psychophysics, 51, 599-606.

Treisman, A. (1991). Search, similarity, and integration of features between and within dimensions. Journal of Experimental Psychology: Human Perception \& Performance, 17, 652-676.

Treisman, A., \& Gormican, S. (1988). Features analysis in early vision: Evidence from search asymmetries. Psychological Review, 95 , 15-48.

Treisman, A., \& Sato, S. (1990). Conjunction search revisited. Journal of Experimental Psychology: Human Perception \& Performance, $16,459-478$.
Tsal, Y., Meiran, N., \& Lamy, D. (1995). Towards a resolution theory of attention. Visual Cognition, 2, 313-330.

Wolfe, J. M. (1994). Guided Search 2.0. A revised model of visual search. Psychonomic Bulletin \& Review, 1, 202-238.

Wolfe, J. M., Cave, K. R., \& Franzel, S. L. (1989). Guided search: An alternative to the feature integration model for visual search. Journal of Experimental Psychology: Human Perception \& Performance, 15, 419-433.

YANTIS, S., \& Egeth, H. E. (in press). Visual salience and stimulusdriven capture. Journal of Experimental Psychology: Human Perception \& Performance.

YANTIS, S., \& JoNIDES, J. (1990). Abrupt visual onsets and selective attention: Voluntary versus automatic allocation. Journal of Experimental Psychology: Human Perception \& Performance, 16, 121-134.

\section{NOTES}

1. In this paper, we shall use the term salience in order to refer to this narrow definition of bottom-up factors.

2. The average RT for the $50 \%$ slowest trials in the target-absent condition was $935 \mathrm{msec}$.

(Manuscript received October 3, 1997; revision accepted for publication March 6, 1998.) 